Review Article

\title{
MOLECULARLY IMPRINTED POLYMER NANOPARTICLES (MIP-NPS) APPLICATIONS IN ELECTROCHEMICAL SENSORS
}

\author{
DIANE FAUZI, FEBRINA AMELIA SAPUTRI*
}

Department of Pharmaceutical Analysis and Medicinal Chemistry, Faculty of Pharmacy, Universitas Padjadjaran, Jl Raya Bandung Sumedang km 21 Jatinangor, West Java, Indonesia, 45363

Email: febrina@unpad.ac.id

Received: 25 Jul 2019, Revised and Accepted: 23 Sep 2019

\begin{abstract}
Molecularly Imprinted Polymers (MIPs) is a polymer that binds together to form a specific binding site that is selective for certain analytes. Its high stability, its synthesize simplicity, and it can ease costs significantly make it was applied widely as a receptor instead of antibodies or enzymes. MIPs can be re-developed into MIPs nanoparticles (MIP-NPs) which have greater potential. MIPs use in electrochemical sensors have relevant applications in daily life and have been tested in human samples. Electrochemical sensors have been successfully functioned with MIP-NPs leading to real-time monitoring of drugs, pesticides, environmental contaminants, and secondary metabolites, as well as molecules with biological relevance. The aim of this review is to summarize the developments and applications of MIP-NPs as a selective recognition component in electrochemical sensors with special emphasis on their analytical applications.
\end{abstract}

Keywords: Electrochemical sensor, Molecularly imprinted polymers, Nanoparticle

(C) 2019 The Authors. Published by Innovare Academic Sciences Pvt Ltd. This is an open access article under the CC BY license (http://creativecommons.org/licenses/by/4.0/) DOI: http://dx.doi.org/10.22159/ijap.2019v11i6.35088

\section{INTRODUCTION}

Mostly, analytical methods require both specific and selective recognition of target analyte. Antibodies, enzymes, and receptors, which are biomolecules, have fulfilled these requirements. Unfortunately, biomolecule use is limited to certain conditions, proteins will be denatured under extreme $\mathrm{pH}$ and temperature, as well as in organic solvent. In addition, preparation and isolation of these biomolecules are time-consuming and their high price makes their use is limited. Therefore, artificial receptors with the same principle as biomolecule receptors are developed. An approach to get these artificial receptors can be through molecularly imprinted polymers (MIPs) [1]. MIPs is a method to generate recognition sites on synthetic polymer for a specific analyte. Artificially, the recognition sites have complement shape, sizes, and chemical functions to the target analyte, make it having specific and selective recognition properties of target analyte [2].

Furthermore, MIPs own several advantages over biomolecule, including high stability, easy to synthesis, and significantly ease the costs [3]. This technique can be applied widely to various analyte, including small molecule (drugs, pesticide, peptide, and sugar), large organic molecule, and bioanalyte (virus, immunoglobulin, and erythrocyte) [1].

However, traditional MIPs synthesis methods produce bulk polymeric microparticles, which are difficult to be integrated into a sensor and in vivo applications, such as medical imaging and drug delivery. Hence, MIPs can be re-developed into MIPs nanoparticles (MIP-NPs), which have greater potential. As known, the properties of nanoscale materials are substantially distinct from bulk matrices due to strong adsorption efficiency, high surface to volume ratio, high surface reactivity, high solubility, better diffusion, and ease of immobilization $[3,4]$.

The number of publications related to the applications of MIP-NP has increased in recent years, especially, in the field of analytical chemistry, such as applications for solid-phase extraction [5, 6], liquid chromatography [7], drug delivery system [8, 9], capillary electrochromatography [10], enzyme-like catalysis [11], and sensor $[12,13]$.

MIPs use in electrochemical sensors have relevant applications in daily life and have been tested in human samples [14]. Nowadays, its development can be used to obtain biochemical information about physiology, metabolism, and disease state. Electrochemical sensors have shown their potential for rapid and real-time measurement of biological molecules [15]. The aim of this review is to summarize the developments and applications of MIP-NPs as a selective recognition component in electrochemical sensors with special emphasis on their analytical applications.

\section{Molecularly imprinted polymers (MIPs)}

Molecularly Imprinted Polymers (MIPs)are a technique to make a polymer with a specific binding site for the specified compound [16]. Fig. 1 shows the scheme of the MIPs formation process. When the template and functional monomers are mixed, a prepolymerization complex is formed which is stable in a predetermined solvent. The polymerization process will occur when the appropriate cross-linker is added. Then, the polymer is milled and sifted until the desired particle size. Finally, the template is removed from the polymer, leaving a cavity that is able to bind analyte selectively [17].

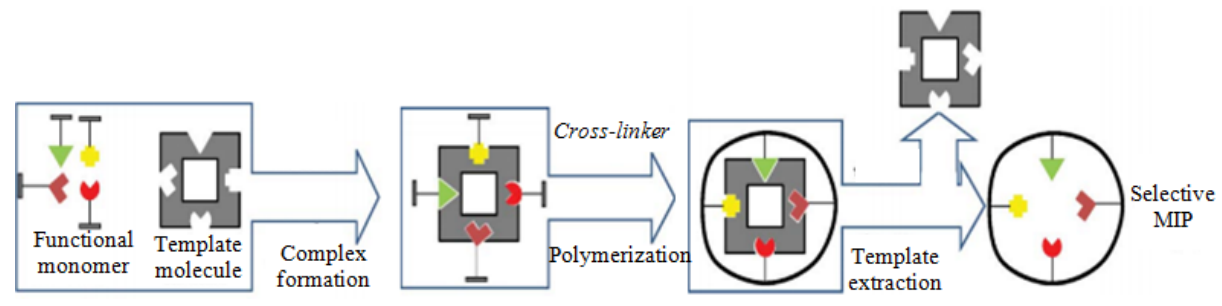

Fig. 1: Schematic representation of the MIPs formation process [18] 
From the previous scheme and explanation, MIPs consist of template, functional monomer, cross-linker, and initiator reaction [19]. Templates are substances that will bind to functional monomers and induce the formation of specific recognition sites in the polymer synthesis process [20]. Meanwhile, functional monomers provide functional groups that will form prepolymerization complexes with templates. Generally, functional monomers have two groups, identification groups that will bind to templates and polymerized groups [21].

Cross-linker has an important role in MIPs synthesis. It controls the polymer matrix morphology, stabilizes the imprinted binding site, and increase mechanical stability to maintain its ability recognizing molecules [22]. To initiate MIPs synthesis, an initiator is needed to release free radicals with unpaired electrons which can react with monomers or cross-linkers easily [23].
All components will be dissolved in a solvent or porogen into one phase in the polymerization process. Besides, solvents also play a role in making pores in the polymer. The formed pores must be large in order resulting MIPs with good flow properties. The more solvents used, the greater pores will be formed [22].

\section{MIPs nanoparticles (MIP-NPs)}

Basically, nanostructured materials are known due to their fundamental properties as quantum sized and have a large surface area. These properties make nanomaterials more advantageous than of other large materials. Thus, it has huge application in biology and medicine for food and drug development $[24,25]$. There are several methods can be used to synthesize imprinted nanoparticles. Fig. 2 shows the various methods and their main advantages.

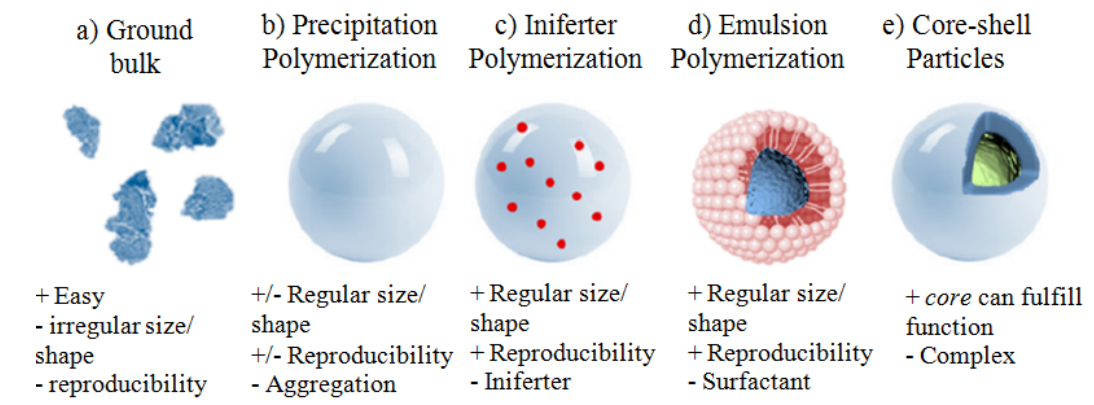

Fig. 2: Comparison of various MIP-NPs synthesis methods [1]

The simplest MIP-NPs synthesis method is the bulk polymerization followed by grinding to get the desired size. However, this method will form particles with irregular size and shape, which will cause reproducibility problems. To obtain particles in desired size can be done by sieving, but this sifting will reduce the yield of the synthesized polymer [22]. Therefore, its use is limited in industry manufacturing standard and production process [26].

Alternative methods provide imprinted polymer in regular size and shape. The other methods, including precipitation polymerization $[27,28]$, emulsion polymerization [29], iniferter polymerization [30], and core-shell particles [31]. Precipitation polymerization gives a better control of shape and size. Iniferter polymerization and emulsion polymerization are other alternatives to improve monodispersity. Iniferter polymerization uses iniferter (red in fig. 2) to stabilize particles at a particular size, while emulsion polymerization uses surfactant. Core-shell particles utilize cores which can have several functions. Then, MIPs is polymerized on the surface of the core [1].

\section{Electrochemical sensors}

Currently, electrochemical sensors are applied widely in various industries. A large number of analytical instruments used in environmental, food, clinical or pharmaceutical labs, as well as most of the commercial care devices run using chemical sensors as a whole or basic part. [32]. Compared to spectrometry (FTIR, UV-VIS), mass spectrometry (MS), and chromatographic techniques (GC, HPLC), electrochemical sensors application are simpler on electronic equipment. Also, maintenance and calibration are easy to do. Sensor signals are given directly (insitu), so they can provide real-time information as process control. Thus, electrochemical sensor is a very elegant method in the pharmaceutical analysis and its application in industry more preferable $[33,34]$. In addition, other advantages of electrochemical sensors are low limit of detection, wide linear range, good stability and reproducibility [32].

Electrochemical sensors are devices that convert electrochemical output into a useful signal for analysis. Generally, electrochemical sensors consist of two main parts, the most crucial part of the sensor is a chemical recognition system and a physicochemical converter device called transducer, which transforms chemical response into detected signal by electrical instruments. These two parts form an electrode (sensor) [32].

Briefly, electrochemical sensor is a device that transforms the interaction between analytes and receptors on the surface of the electrode into a measurable analytical signal. Electrochemical sensor can use a different electroanalytical technique, commonly used measurement principles are currents with varying potential (voltammetry), current at fixed voltage (amperometry), voltage at zero current (potentiometry), conductivity, and changes in capacitance or impedance [14].

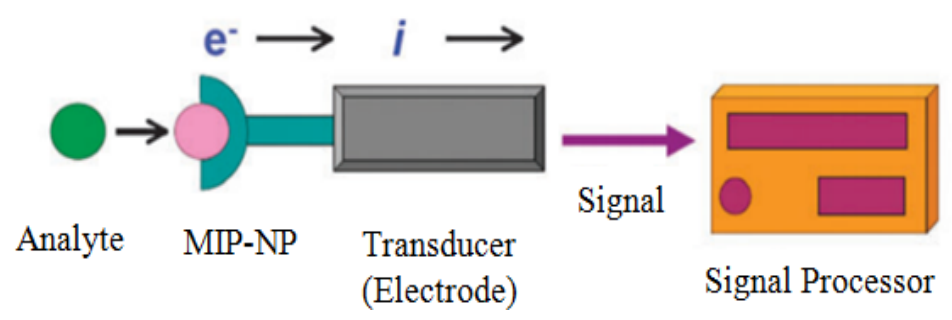

Fig. 3: Schematic representation of MIP-NP application in electrochemical sensors [34] 


\section{MIP-NPs in electrochemical sensors}

MIPs has been applied in various aspects of chemical analysis, including electrode modification. The major purpose of this modification is to increase selectivity. Besides, an increase sensitivity also occurs in various cases. In all cases, various analytes, including drugs, pesticides, environmental contaminants, and secondary metabolites, as well as molecules with biological relevance have been successfully used as templates and analyzed by electrochemical devices in electroanalytic procedures [35].

The schematic representation of the application of MIP-NP in electrochemical sensors is shown in fig. 3. This device consists of MIP-NP, transducer or electrode, and signal processor. The transducer is modified by adding MIP-NP onto its surface as a recognition element of the target analyte, which will increase sensitivity and selectivity by enhanced analytical response for the quantification. When MIP-NP binds to the target analyte, the transducer will produce electrical signal depend on the concentration or number of target analytes that are bind to MIP-NP. Then, the signal was measured by the signal processor electrochemically, either through voltammetry, amperometry, potentiometry, or impedance [14, 36-38].

Electrochemical sensors based on MIP-NPs for drug analysis have been developed using various analytes, such as erythromycin, levofloxacin, losartan, metronidazole, tetracycline, tramadol, and trazosin. All of them were developed using different sensors and different types of cores with polymerized MIP on its surface as shown in table 1.

Table 1: Summary of some MIP-NPs applications in electrochemical sensing

\begin{tabular}{|c|c|c|c|c|c|c|}
\hline Analyte & $\begin{array}{l}\text { Polymerization } \\
\text { method }\end{array}$ & Sensors & $\begin{array}{l}\text { Detection } \\
\text { limit (M) }\end{array}$ & Linear range (M) & Matrix & Reference \\
\hline $\begin{array}{l}\text { 2,4-dichlorophenol } \\
(2,4-D C P)\end{array}$ & Core-shell & SWV & $10^{-8}$ & $0.04-2 \times 10^{-6}$ & Water & [39] \\
\hline $17 \beta$-estradiol & Core-shell & DPV & $1.6 \times 10^{-8}$ & $3 \times 10^{-8}-5 \times 10^{-5}$ & Water & [38] \\
\hline Aflatoxin B1 & Core-shell & LSV & $0.3 \mathrm{fg} / \mathrm{ml}$ & $1 \mathrm{fg} / \mathrm{ml}-1 \mu \mathrm{g} / \mathrm{ml}$ & - & {$[40]$} \\
\hline Bisphenol A (BPA) & Core-shell & Amperometry & $1.38 \times 10^{-7}$ & $8 \times 10^{-6}-6 \times 10^{-2}$ & Bottle Water & [41] \\
\hline Chlortoluron & Core-shell & $\mathrm{CV}$ & $2.4 \times 10^{-9}$ & $10^{-8}-10^{-4}$ & Water & [12] \\
\hline Cholesterol & Core-shell & CV and DPV & $3.3 \times 10^{-}[14]$ & $10^{-}[13]-10^{-9}$ & - & [42] \\
\hline Diazinon & $\begin{array}{l}\text { Suspension } \\
\text { Polymerization }\end{array}$ & CV and SWV & $7.9 \times 10^{-}[10]$ & $\begin{array}{l}2.5 \times 10^{-9}-1 \times 10^{-7} \text { and } \\
1 \times 10^{-7}-2 \times 10^{-6}\end{array}$ & Apple and Water & [43] \\
\hline Dibutyl phthalate & Core-shell & DPV & $8 \times 10^{-}[10]$ & $2.5 \times 10^{-9}-5 \times 10^{-6}$ & Wine & [44] \\
\hline Diphenylamine & Core-shell & DPV & $0.05 \times 10^{-6}$ & $0.1-30 \times 10^{-6}$ & Lake Water & [45] \\
\hline Dopamin & Core-shell & DPV & $3 \times 10^{-8}$ & $5 \times 10^{-8}-1.6 \times 10^{-4}$ & Urine & [46] \\
\hline Dopamin & Core-shell & Potentiometry & $1 \times 10-9$ & $10^{-9}-10^{-6}$ & Blood serum and urine & [47] \\
\hline Dopamin & Core-shell & $\mathrm{CV}$ & $7.63 \times 10^{-}[14]$ & $2 \times 10^{-}[13]-2 \times 10^{-8}$ & $\begin{array}{l}\text { Rabbit blood and rat } \\
\text { brain tissue }\end{array}$ & [48] \\
\hline Dopamin & Core-shell & $\mathrm{CV}$ & $2 \times 10^{-8}$ & $4.8 \times 10^{-8}-5 \times 10^{-5}$ & $\begin{array}{l}\text { Pharmaceutical } \\
\text { product and urine }\end{array}$ & [49] \\
\hline Erythromycin & Core-shell & Amperometry & $2.3 \times 10^{-8}$ & $7 \times 10^{-8}-9 \times 10^{-5}$ & Milk and honey & {$[50]$} \\
\hline Estrone & $\begin{array}{l}\text { Emulsion } \\
\text { Polymerization }\end{array}$ & EIS & - & - & - & [51] \\
\hline Histamine & $\begin{array}{l}\text { Iniferter } \\
\text { Polymerization }\end{array}$ & Potentiometry & $1.2 \times 10^{-6}$ & $10^{-6}-10^{-2}$ & Wine and fish & {$[52]$} \\
\hline Levofloxacin & Core-shell & DPV & $0.53 \times 10^{-6}$ & $1-100 \times 10^{-6}$ & Capsule & [53] \\
\hline Losartan & Core-shell & Potentiometry & $1.82 \times 10^{-9}$ & $3 \times 10^{-9}-1 \times 10^{-2}$ & $\begin{array}{l}\text { Pharmaceutical } \\
\text { product and urine }\end{array}$ & [54] \\
\hline Metronidazole & Core-shell & $\mathrm{CV}$ & $1.8 \times 10^{-[11]}$ & $\begin{array}{l}5 \times 10^{-}[11]-1 \times 10^{-9} \\
\text { and } 1 \times 10^{-9}-1.4 \times 10^{-6}\end{array}$ & Fish tissue & [55] \\
\hline Octylphenol & Core-shell & LSV & $\begin{array}{l}6 \times 10^{-9} \text { and } 1 \\
\times 10^{-9}\end{array}$ & $\begin{array}{l}0.04-8 \times 10^{-6} \text { and } \\
0, .02-8 \times 10^{-6}\end{array}$ & Water and urine & [56] \\
\hline $\mathrm{Pb}$ (II) ion & $\begin{array}{l}\text { Precipitation } \\
\text { Polymerization }\end{array}$ & DPV & $10^{-9}-10^{-6}$ & $10-[12]$ & Water & [57] \\
\hline Quercetin & Core-shell & DPV & $4.8 \times 10^{-8}$ & $6 \times 10^{-7}-1.5 \times 10^{-5}$ & Apple juice & [58] \\
\hline Ractopamine & Core-shell & DPV & $0.02 \times 10^{-6}$ & $0.002-0.1 \times 10^{-6}$ & Water & [59] \\
\hline Tetracycline & Core-shell & CV and EIS & $0.04 \mathrm{mg} / \mathrm{l}$ & $0.1-30 \mathrm{mg} / \mathrm{l}$ & - & [60] \\
\hline Tramadol & Core-shell & SWV & $0.004 \times 10^{-6}$ & $0.01-20 \times 10^{-6}$ & $\begin{array}{l}\text { Pharmaceutical } \\
\text { product and urine }\end{array}$ & [61] \\
\hline Trazosin & Core-shell & DPV & $0.3 \times 10^{-6}$ & $2-150 \times 10^{-6}$ & $\begin{array}{l}\text { Blood serum, urine, } \\
\text { tablet }\end{array}$ & [62] \\
\hline Tyrosin & Core-shell & DPV & $1.5 \times 10^{-}[10]$ & $1 \times 10^{-9}-2 \times 10^{-8}$ & Milk & [63] \\
\hline
\end{tabular}

Note: SWV = Square Wave Voltammetry; DPV = Differential Pulse Voltammetry; LSV = Linear Sweep Voltammetry; CV = Cyclic Voltammetry; EIS = Electrochemical Impendance Spectroscopy

In 2011, Wang et al. (2011) reported tetracycline (TC) sensors formed from MIP and gold nanoparticles (AuNPs) modified with multiwall carbon nanotubes (MWNTs-GNPs) [60]. Then, erythromycin sensors were developed by Lian et al. (2012), which used gold electrodes with chitosan-platinum nanoparticles (CS$\mathrm{PtNP}$ ) and graphene-gold nanoparticles (GR-AuNP). The synergistic effect of CS-PtNP and GR-AuNP increases electrochemical response and sensor sensitivity [50].

Meanwhile, Wang et al. (2014) only use GR-AuNP for levofloxacin sensors. In this sensor, GR-AuNPs encourages levofloxacin electroxidation on the electrode, while molecularly imprinted levoflocaxin polypyrrole serves as an element of recognition [53]. Previously, Afkhami et al. (2013) developed a sensor for tramadol by synthesizing nano-MIP from SiO2 @ Fe304 and applying it to carbon paste electrodes (CPE). Sensors with chemically modified CPE have been used for tramadol determination in healthy human urine and pharmaceutical samples [61].

Then, losartan sensors was developed by Bagheri et al. (2015), MIP was synthesized and used as a recognition tool on modified nanographene CPE. The developed potentiometric sensor showed a fast response time of $\sim 6 \mathrm{~s}$, high performance, and high selectivity on some interference compounds, also satisfying long-term stability ( $>3$ 
mo). This sensor was successfully applied to measurements of losartan sensitvely in urine and pharmaceutical samples with satisfying results [54].

Metronidazole sensor was developed by Li et al. (2015), it uses a nanoporous gold leaf (NPGL) as a loading platform for MIP immobilization, which have a large surface area that can be passed by superb electrical conductivity [55]. Then, trazosin (TR) sensor was developed by Roushani et al. (2018), voltammetric sensors are made based on MIP and AuNPs modified with carbon electrodes (MIP/AuNP/SPCE). These sensors selectively detect TR even in the presence of similar compounds with high concentrations and MIP/AuNP/SPCE also successfully determined TR in several real samples, including human urine, blood serum, and trazosin tablets [62].

Aside from drugs, electrochemical sensors based on MIP-NP are also developed for pesticide determination, such as chlortoluron and diazonin. Li et al. (2013) designed a chlortoluron sensor with MIP synthesized on the surface of magnetic nickel (II) oxide (NiO) nanoparticles, which based on change in $\mathrm{H}_{2} \mathrm{O}_{2}$ oxidation current. Chlrotoluron can be indirectly analyzed by decreasing the oxidation current of $\mathrm{H} 2 \mathrm{O} 2$ on the glass carbon electrode (GCE) modified by $\mathrm{NiO}$ nanoparticles due to blocking access after rebinding. NiO nanoparticles provide high catalytic effect on $\mathrm{H} 2 \mathrm{O} 2$ oxidation resulting high sensitivity [12]. Another case, Motaharian et al. (2016) developed diazinone sensor (DZN) by synthesizing diazinone MIP nanoparticles through suspension polymerization and then used it as part of CPE modification [43].

In addition, the application of MIP-NP in electrochemical sensors was also used to detect contaminant compounds in the environment. Some analytes in the form of environmental contaminants are 2,4dichlorophenol (2,4-DCP), $17 \beta$-estradiol (E2), aflatoxin B1 (AFB1), bisphenol A (BPA), dibutyl phthalate (DBP), diphenylamine, $\mathrm{Pb}$ (II) ions, octylphenil, and ractopamine.

Liu et al. (2016) designed an electrochemical sensor based on MIPNP which was made through pyrrole electropolymerization on GCE modified Fe304 nanoparticles. The sensor showed high catalysis ability to oxidize 2,4-DCP [39]. Meanwhile, the electrochemical sensor for 17ß-estradiol (E2) was developed by Yuan et al. (2011), it based on MIP by forming 6-mercaptonicotinic acid (MNA) and E2 polymer membranes through electropolymerization on the surface of modified GCE platinum nanoparticles (PtNPs/GCE). In his research, showed that the sensor is effective for real-time determining of E2 in a complicated matrix because of its large adsorption capacity and high selectivity [38].

Then, Jiang et al. (2015) designed an electrochemical sensor with electropolymerization of $\mathrm{p}$-aminothiophenol gold nanoparticles with aflatoxin B1 (AFB1) as a template molecule [40]. Huang et al. (2011) developed a bisphenol A (BPA) sensor with amperometric detection using MIP and AuNPs. The susceptible layer was synthesized via 2aminothiophenol electropolymerization on GCE-AuNP with a BPA template [41].

Li et al. (2015) developed the dibutyl phthalate electrochemical sensor (DBP) with molecular recognition elements from magnetic graphene oxide @ gold nanoparticle-MIP (MGO @ AuNP-MIP) [44]. Different case with Bojdi et al. (2014), who developed an electrochemical sensor to detect $\mathrm{Pb}$ (II) ions using depositional polymerization techniques [57].

Previously, MIP and AuNP-based electrochemical sensors also have been developed by $\mathrm{Li}$ et al. (2018) to selectively monitor ractopamine (RAC) in water. This sensor consists of nanobead $\mathrm{Fe} 304$ and AuNP on the substrate of reduced graphene oxide (RGO), was made using the reversible addition fragmentation chain transfer (RAFT) polymerization technique. The study shows the potential of the electrochemical sensors in monitoring organic pollutants in water [59].

The application of MIP-NP in electrochemical sensors also can be applied for secondary metabolites detection, for example quercetin. MIP-based polypyrrole film with graphene oxide was made and used for the determination of electrochemical quercetin by Sun et al.
(2013). These electrodes showed great stability and reproducibility. Rutin or morin, which has the same structure as quercetin, at the same concentration did not interfere quercetin determination. The application of this methods for complex matrix analysis was also evaluated in apple juice samples [58].

Then, electrochemical sensors based on MIP-NP was used to detect biological molecules, such as cholesterol, dopamine, estrone, histamine, and tyrosine. Ji et al. (2015) developed electrochemical sensors for cholesterol detection by synthesizing MIP on modified multi-walled carbon nanotubes (MWCNT) and AuNP. pAminothiophenol (P-ATP) and CHO converge on the surface of the modified GCE forming Au-S bonds and hydrogen bonds interaction, and the polymeric membrane was formed by electropolymerization. The developed system has a potential for application in clinical diagnosis of cholesterol with high-speed real-time detection capability, slight sample compsumption, high sensitivity, low interference, and good stability [42].

The development of dopamine sensors was carried out by four researchers. They used core-shell particle to fabricate the MIP-NP. Yu et al. (2012) used graphene oxide (GO) and Zeng et al. (2013) used AuNP as a core coated with $\mathrm{SiO} 2$ and MIP, which was synthesized by the sol-gel technique [46,49]. Then, Li et al. (2016) developed metallic microrod electrodes namely, nanoporous $\mathrm{Au}-\mathrm{Ag}$ alloy microrod (NPAMR) and modified with electropolymerization MIP [48]. Meanwhile, Anirudhan et al. (2014) used MWCNTs-MIP for dopamine determination in human urine and blood serum samples with detection limit of $1.0 \times 10^{-9} \mathrm{M}$ and response time of $\sim 2 \mathrm{~min}$ means the developed sensors can be considered as a sensitive tool for detection of dopamine depletion in Parkinson's disease. This sensor showed high sensitivity, high selectivity, high stability, good reproductivity, and long-term useful lifetime $(>2 \mathrm{mo})$. This sensor was successfully evaluated in real samples, blood serum, and urine samples [47].

Yola et al. (2015) developed electrochemical sensors using cubic AuNP (cAuNP) on GCE for tyrosine determination [63]. Congur et al. (2013) designed an electrochemical sensor using MIP-NP, which was synthesized by free surfactant emulsion polymerization method, and produced MIP-NP with $163.2 \mathrm{~nm}$ diameter, which served as a recognition element to determine estrone using impedance spectroscopy [51]. Then, synthesis MIP-NP through solid phase printing was carried out by Basozabal et al. (2014) for detection histamine by potentiometry. This MIP-NP was inserted into a polyvinylchloride (PVC) membrane to make a selective histamine electrode in a real sample, including wine and fish [52].

Increasing the number of studies in the field of electrochemical sensors based on MIP-NP indicates that interest in this field is developing continuously. These sensors are interesting because its specificity, selectivity, cost effective, physically and chemically stable compared to biomolecules, ease preparation, and can be applied to various molecules. This sensor system is expected to develop rapidly and continuously, especially in the pharmaceutical and medical fields, as well as other fields. In the future, these sensors can be developed in the form of handheld devices. It will let patients check and see the results by themselves without any medical assistance. This sensor type will revolutionize health care industry by reducing maintenance costs and improving clinical outcomes [64].

\section{CONCLUSION}

The combination of electrochemical sensors and MIP-NP layers, as identification elements, is a promising approach for the development of biomimetic sensors that are able to overcome the limitations of traditional biosensors, such as easily denatured at extreme temperatures and $\mathrm{pH}$, as well as in organic solvents. The electrochemical sensor has been successfully functioned with MIP$\mathrm{NP}$, which leads to a real-time monitoring of drugs, pesticides, environmental contaminants, and secondary metabolites, as well as molecules with biological relevance. This sensor is effective in terms of cost and simple preparation, and the possibility of being developed in the form of a handheld device, also its fast response allows for detection and quantification in just a few minutes or even seconds. 


\section{AUTHORS CONTRIBUTIONS}

All the authors have contributed equally

\section{CONFLICT OF INTERESTS}

\section{Declared none}

\section{REFERENCES}

1. Wackerlig J, Schirhagl R. Applications of molecularly imprinted polymer nanoparticles and their advances toward industrial use: a review. Anal Chem 2016;88:250-61.

2. Belbruno JJ. Molecularly imprinted polymers. Chem Rev 2019;119:94-119.

3. Subrahmanyam S, Guerreiro A, Poma A, Moczko E, Piletska E, Piletsky S. Optimisation of experimental conditions for synthesis of high affinity MIP nanoparticles. Eur Polym J 2013;49:100-5

4. Mustafa G, Lieberzeit PA. Molecularly imprinted polymer-Ag2S nanoparticle composites for sensing volatile organics. RSC Adv 2014;4:12723-8.

5. Farzaneh S, Asadi E, Abdouss M, Barghi Lish A, Azodi Deilami S, Khonakdar HA, et al. Molecularly imprinted polymer nanoparticles for olanzapine recognition: application for solid phase extraction and sustained release. RSC Adv 2015;5:9154-66.

6. Omidi F, Behbahani M, Abandansari HS, Sedighi A, Shahtaheri SJ. Application of molecular imprinted polymer nanoparticles as a selective solid phase extraction for preconcentration and trace determination of 2,4-dichlorophenoxyacetic acid in the human urine and different water samples. J Environ Heal Sci Eng 2014;12:137.

7. Attallah OA, Al-Ghobashy MA, Ayoub AT, Nebsen M. Magnetic molecularly imprinted polymer nanoparticles for simultaneous extraction and determination of 6-mercaptopurine and its active metabolite thioguanine in human plasma. J Chromatogr A 2018;1561:28-8.

8. Bakhshizadeh M, Sazgarnia A, Seifi M, Hadizadeh F, Rajabzadeh G, Mohajeri SA. TiO2-based mitoxantrone imprinted poly (Methacrylic acid-co-polycaprolctone diacrylate) nanoparticles as a drug delivery system. Curr Pharm Des 2017;23:2685-94.

9. Esfandyari Manesh M, Darvishi B, Ishkuh FA, Shahmoradi E, Mohammadi A, Javanbakht M, et al. Paclitaxel molecularly imprinted polymer-PEG-folate nanoparticles for targeting anticancer delivery: characterization and cellular cytotoxicity. Mater Sci Eng C 2016;62:626-33.

10. Liu X, Zong HY, Huang YP, Liu ZS. Liquid crystal-based molecularly imprinted nanoparticles with low crosslinking for capillary electrochromatography. J Chromatogr A 2013;1309:84-9.

11. Hu L, Zhao Y. Molecularly imprinted artificial esterases with highly specific active sites and precisely installed catalytic groups. Org Biomol Chem 2018;16:5580-4.

12. Li X, Zhang L, Wei $\mathrm{X}$, Li J. A sensitive and renewable chlortoluron molecularly imprinted polymer sensor based on the gate-controlled catalytic electrooxidation of $\mathrm{H} 2 \mathrm{O} 2$ on magnetic nano-NiO. Electroanalysis 2013;25:1286-93.

13. Lian HT, Liu B, Chen YP, Sun XY. A urea electrochemical sensor based on molecularly imprinted chitosan film doping with CdS quantum dots. Anal Biochem 2012;426:40-26.

14. Ahmad OS, Bedwell TS, Esen C, Garcia Cruz A, Piletsky SA. Molecularly imprinted polymers in electrochemical and optical sensors. Trends Biotechnol 2019;37:294-309.

15. Dumitrescu E, Andreescu S. Bioapplications of electrochemical sensors and biosensors. Methods Enzymol 1st ed 2017;589:301-50.

16. Hasanah AN, Yulianti AB, Rahayu D. Selective atenolol determination in blood using molecular imprinted polymer with itaconic acid as functional monomer. Int J Appl Pharm 2019;11:136-43.

17. Yi LX, Fang R, Chen GH. Molecularly imprinted solid-phase extraction in the analysis of agrochemicals. J Chromatogr Sci 2013;51:608-18.

18. Płotka Wasylka J, Marc M, Szczepanska N, Namiesnik J. New polymeric materials for solid-phase extraction. Crit Rev Anal Chem 2017;47:373-83.
19. Damayanti S, Gunawan U, Ibrahim S. Development of molecular imprinted polymer solid phase extraction for separation nitrofurantoin residue in chicken eggs. Asian J Pharm Clin Res 2017;10:108.

20. Walsh R. Development and characterisation of molecularly imprinted suspension polymers. Waterford Institute of Technology; 2010.

21. Chen L, Wang X, Lu W, Wu X, Li J. Molecular imprinting: perspectives and applications. Chem Soc Rev 2016;45:2137211.

22. Vasapollo G, Sole R Del, Mergola L, Lazzoi MR, Scardino A, Scorrano $S$, et al. Molecularly imprinted polymers: present and future prospective. Int J Mol Sci 2011;12:5908-45.

23. Rane J, Adhikar P, Bakal RL. Molecular imprinting: an emerging technology. Asian J Pharm Technol Innov 2015;3:2015.

24. Banjare J. Application of nanotechnology in food technology and targeted drug therapy for prevention of obesity: an overview. J Crit Rev 2017;4:7.

25. Dhull V, Dilbaghi N, Hooda V. Nanomaterials based acetylcholinesterase biosensors for organophosphorus compounds detection: review. Int J Pharm Pharm Sci 2015;7:17-24.

26. Moczko E, Poma A, Guerreiro A, Perez De Vargas Sansalvador I, Caygill S, Canfarotta F, et al. Surface-modified multifunctional MIP nanoparticles. Nanoscale 2013;5:3733-41.

27. Liu Y, Zhang G, Deng L, Lei J, Wang L, He J. Synthesis, characterization and adsorption performance of molecularly imprinted nanoparticles for tripterine by precipitation polymerization. Anal Methods 2014;6:684-9.

28. Phungpanya C, Chaipuang A, Machan T, Watla Iad K, Thongpoon C, Suwantong O. Synthesis of prednisolone molecularly imprinted polymer nanoparticles by precipitation polymerization. Polym Adv Technol 2018;29:3075-84.

29. Esfandyari Manesh M, Javanbakht M, Dinarvand R, Atyabi F. Molecularly imprinted nanoparticles prepared by miniemulsion polymerization as selective receptors and new carriers for the sustained release of carbamazepine. J Mater Sci Mater Med 2012;23:963-72.

30. Guerreiro AR, Chianella I, Piletska E, Whitcombe MJ, Piletsky SA. Selection of imprinted nanoparticles by affinity chromatography. Biosens Bioelectron 2009;24:2740-3.

31. Xu J, Haupt K, Tse Sum Bui B. Core-shell molecularly imprinted polymer nanoparticles as synthetic antibodies in a sandwich fluoroimmunoassay for trypsin determination in human serum. ACS Appl Mater Interfaces 2017;9:24476-83.

32. Faridbod F, Gupta VK, Zamani HA. Electrochemical sensors and biosensors. Int J Electrochem 2011;2011:1-2.

33. Hendawy HAM, Elwy HM, Fekry AM. Electrochemical and chemometric determination of dorzolamide and timolol in eye drops using modified multiwall carbon nanotube electrode. Int J Pharm Pharm Sci 2017;9:43.

34. Ronkainen NJ, Halsall HB, Heineman WR. Electrochemical biosensors. Chem Soc Rev 2010;39:1747-63.

35. Cervini P, Cavalheiro ETG. Strategies for preparation of molecularly imprinted polymers modified electrodes and their application in electroanalysis: a review. Anal Lett 2012;45:297-313.

36. Suresh E, Sundaram K, Kavitha B, Kumar NS. Electroanalysis of ibuprofen on conducting polyaniline nanofiber coated glassy carbon surface. Int J Curr Pharm Res 2016;8:44-8.

37. Lahcen AA, Amine A. Recent advances in electrochemical sensors based on molecularly imprinted polymers and nanomaterials. Electroanalysis 2019;31:188-201.

38. Yuan L, Zhang J, Zhou P, Chen J, Wang R, Wen T, et al. Electrochemical sensor based on molecularly imprinted membranes at platinum nanoparticles-modified electrode for determination of $17 \beta$-estradiol. Biosens Bioelectron 2011;29:29-33

39. Liu B, Cang H, Jin J. Molecularly imprinted polymers based electrochemical sensor for 2,4-dichlorophenol determination. Polymers (Basel) 2016;8:309.

40. Jiang M, Braiek M, Florea A, Chrouda A, Farre C, Bonhomme A, et al. Aflatoxin B1 detection using a highly-sensitive molecularly-imprinted electrochemical sensor based on an 
electropolymerized metal-organic framework. Toxins (Basel) 2015;7:3540-53.

41. Huang J, Zhang X, Liu S, Lin Q, He X, Xing X, et al. Electrochemical sensor for bisphenol a detection based on molecularly imprinted polymers and gold nanoparticles. J Appl Electrochem 2011;41:1323-8.

42. Ji J, Zhou Z, Zhao X, Sun J, Sun X. Electrochemical sensor based on molecularly imprinted film at $\mathrm{Au}$ nanoparticles-carbon nanotubes modified electrode for determination of cholesterol. Biosens Bioelectron 2015;66:590-5.

43. Motaharian A, Motaharian F, Abnous K, Hosseini MRM, Hassanzadeh Khayyat M. Molecularly imprinted polymer nanoparticles-based electrochemical sensor for determination of diazinon pesticide in well water and apple fruit samples. Anal Bioanal Chem 2016;408:6769-79.

44. Li X, Wang X, Li L, Duan H, Luo C. Electrochemical sensor based on magnetic graphene oxide@gold nanoparticles-molecular imprinted polymers for determination of dibutyl phthalate. Talanta 2015;131:354-60.

45. Liu L, Zhu X, Zeng Y, Wang $\mathrm{H}$, Lu Y, Zhang J, et al. An electrochemical sensor for diphenylamine detection based on reduced graphene oxide/Fe 30 4-molecularly imprinted polymer with 1,4-butanediyl-3,3'-bis-l-vinylimidazolium dihexafluorophosphate ionic liquid as cross-linker. Polymers (Basel) 2018;10:1329-42.

46. Zeng Y, Zhou Y, Kong L, Zhou T, Shi G. A novel composite of SiO2-coated graphene oxide and molecularly imprinted polymers for electrochemical sensing dopamine. Biosens Bioelectron 2013;45:25-33.

47. Anirudhan TS, Alexander S, Lilly A. Surface modified multiwalled carbon nanotube-based molecularly imprinted polymer for the sensing of dopamine in real samples using potentiometric method. Polymer (Guildf) 2014;55:4820-31.

48. Li Y, Song H, Zhang L, Zuo P, Ye B ce, Yao J, et al. Supportless electrochemical sensor based on molecularly imprinted polymer-modified nanoporous microrod for determination of dopamine at trace level. Biosens Bioelectron 2016;78:308-14.

49. Yu D, Zeng Y, Qi Y, Zhou T, Shi G. A novel electrochemical sensor for determination of dopamine based on AuNPs@SiO2 coreshell imprinted composite. Biosens Bioelectron 2012;38:270-7.

50. Lian W, Liu S, Yu J, Xing X, Li J, Cui M, et al. Electrochemical sensor based on gold nanoparticles fabricated molecularly imprinted polymer film at chitosan-platinum nanoparticles/ graphene-gold nanoparticles double nanocomposites modified electrode for detection of erythromycin. Biosens Bioelectron 2012;38:163-9.

51. Congur G, Senay H, Turkcan C, Canavar E, Erdem A, Akgol S. Estrone specific molecularly imprinted polymeric nanospheres: synthesis, characterization and applications for electrochemical sensor development. Comb Chem High Throughput Screen 2013;16:503-10.

52. Basozabal I, Guerreiro A, Gomez Caballero A, Aranzazu Goicolea M, Barrio RJ. Direct potentiometric quantification of histamine using solid-phase imprinted nanoparticles as recognition elements. Biosens Bioelectron 2014;58:138-44.

53. Wang F, Zhu L, Zhang J. Electrochemical sensor for levofloxacin based on molecularly imprinted polypyrrole-graphene-gold nanoparticles modified electrode. Sensors Actuators B Chem 2014;192:642-7.

54. Bagheri H, Shirzadmehr A, Rezaei M. Designing and fabrication of new molecularly imprinted polymer-based potentiometric nano-graphene/ionic liquid/carbon paste electrode for the determination of losartan. J Mol Liq 2015;212:96-102.

55. Li Y, Liu Y, Liu J, Liu J, Tang H, Cao C, et al. Molecularly imprinted polymer decorated nanoporous gold for highly selective and sensitive electrochemical sensors. Sci Rep 2015;5:33-5.

56. Pan Y, Zhao F, Zeng B. Electrochemical sensors of octylphenol based on molecularly imprinted poly(3,4-ethylenedioxythiophene) and poly(3,4-ethylenedioxythiophene-gold nanoparticles). RSC Adv 2015;5:57671-7.

57. Bojdi MK, Mashhadizadeh MH, Behbahani M, Farahani A, Davarani SSH, Bagheri A. Synthesis, characterization and application of novel lead imprinted polymer nanoparticles as a high selective electrochemical sensor for ultra-trace determination of lead ions in complex matrixes. Electrochim Acta 2014;136:59-65.

58. Sun S, Zhang M, Li Y, He X. A molecularly imprinted polymer with incorporated graphene oxide for electrochemical determination of quercetin. Sensors (Switzerland) 2013;13:5493-506.

59. Li Y, Xu W, Zhao X, Huang Y, Kang J, Qi Q, et al. Electrochemical sensors based on molecularly imprinted polymers on Fe 30 4/graphene modified by gold nanoparticles for highly selective and sensitive detection of trace ractopamine in water. Analyst 2018;143:5094-102.

60. Wang $\mathrm{H}$, Zhao $\mathrm{H}$, Quan $\mathrm{X}$, Chen S. Electrochemical determination of tetracycline using molecularly imprinted polymer-modified carbon nanotube-gold nanoparticles electrode. Electroanalysis 2011;23:1863-9.

61. Afkhami A, Ghaedi H, Madrakian T, Ahmadi M, Mahmood Kashani H. Fabrication of a new electrochemical sensor based on a new nano-molecularly imprinted polymer for highly selective and sensitive determination of tramadol in human urine samples. Biosens Bioelectron 2013;44:34-40.

62. Roushani M, Jalilian Z, Nezhadali A. A novel electrochemical sensor based on electrode modified with gold nanoparticles and molecularly imprinted polymer for rapid determination of trazosin. Colloids Surf B 2018;172:594-600.

63. Yola ML, Eren T, Atar N. A sensitive molecular imprinted electrochemical sensor based on gold nanoparticles decorated graphene oxide: application to the selective determination of tyrosine in milk. Sensors Actuators, B Chem 2015;210:149-57.

64. Saylan Y, Akgonullu S, Yavuz H, Unal S, Denizli A. Molecularly imprinted polymer-based sensors for medical applications. Sensors (Switzerland) 2019;19:1279-98. 